\title{
Adaptación y validación de la Escala de Intenciones Futuras de Comportamiento en usuarios de servicios deportivos*
}

\author{
Adaptation and Validation of the Scale of Future Intentions \\ of Behaviour in Customers of Sport Services
}

Recibido: octubre 15 de 2012 | Revisado: febrero 12 de 2014 | Aceptado: febrero 12 de 2014

\author{
Alberto Nuviala Nuviala** \\ JERÓNIMO GARCÍA-FERNÁNDEZ *** \\ AINARA BERNAL-GARCÍA ***** \\ Universidad de Sevilla, España \\ Alberto GraO-CRUCES ****** \\ MARÍA RosARIO TEVA-VILLÉN ******* \\ RAQUEL PÉREZ-ORDÁS ******** \\ Universidad Pablo de Olavide, España
}

doi:10.11144/Javeriana.UPSY13-3.avei

Para citar este artículo: Nuviala, A., GarcíaFernández, J., Bernal-García, A., Grao-Cruces, A., Teva-Villén, M. R., \& Pérez-Ordás, R. (2014). Adaptación y validación de la Escala de Intenciones Futuras de Comportamiento en usuarios de servicios deportivos. Universitas Psychologica, 13(3), 1071-1082. http://dx.doi.org/10.11144/Javeriana. UPSY13-3.avei

Artículo original resultado de investigación.

** Profesor Titular de Universidad. Correo electrónico: anuvnuv@upo.es

*** Correo electrónico: agracru@upo.es

***** Profesor Asociado. Correo electrónico: rteva@ upo.es

******* Profesor Contratado. Correo electrónico: rperord@upo.es

********* Profesor Contratado. Doctor. Correo electrónico: jeronimo@us.es

*********** Correo electrónico: abernal1@us.es

\section{RESUMEN}

La fidelización de usuarios en cualquier tipo de organización es un tema transcendente por la cascada de beneficios que se producen al incrementarla. Las organizaciones deportivas no son ajenas a esta realidad. El objetivo de este estudio fue adaptar y validar una escala de intenciones futuras de comportamiento a servicios deportivos. Para la validación de la misma, se utilizó una muestra compuesta por 2181 usuarios. Se realizó un análisis factorial confirmatorio precedido del exploratorio. Se calculó la fiabilidad del instrumento resultante y su validez discriminante. Los resultados permiten afirmar que se dispone de una herramienta válida y fiable compuesta por tres dimensiones y diez ítems, capaz de conocer las intenciones futuras de comportamiento de usuarios de servicios deportivos.

Palabras clave

Validez; fiabilidad; fidelización; servicios deportivos

\section{A B S T R A C T}

Customer loyalty, inside any organization, is transcendental because of the reported income it delivers. Sport business is no exception. This research's goal was to set up and validate a scale of future intentions of behaviour in sport services. A sample of 2181 users was used in order to validate this scale. Factorial exploratory and then confirmatory analyses were made. Reliability and discriminatory validity of the resulting tool were calculated. Results show a reliable and valid tool with three dimensions and ten items, able to determine future intentions of behaviour of sport services customers. Keywords

Validity; reliability; loyalty; sport services 
Alberto Nuviala Nuviala, Alberto Grao-Cruces, María Rosario Teva-Villén, Raquel Pérez-Ordás, Jerónimo García-Fernández, Ainara Bernal-García

\section{Introducción}

Fidelizar a los clientes supone muchos beneficios, económicos y no económicos, por lo que la implantación y mejora continuada de los programas de lealtad se están convirtiendo en una estrategia para maximizar los beneficios de las empresas (Chen \& Quester, 2006). La definición más habitual de fidelidad es la propuesta por Oliver (1999) que la entiende como el compromiso de recomprar un producto/servicio en el futuro, provocando así repetidas compras de la misma marca, pese a que las influencias situacionales y los esfuerzos de marketing tengan el potencial de generar un cambio de comportamiento.

Existen tres enfoques relativos a la lealtad del cliente (Jacoby \& Chestnut, 1978): enfoque conductual, enfoque actitudinal e integración de ambos enfoques. El primero, también denominado lealtad como comportamiento, la concibe como la repetición de compra de una determinada marca (Buttle \& Burton, 2002), entendiéndose como único indicador. En esta perspectiva, se identifica una limitación importante, al entender la lealtad desde un sentido comportamental. Las empresas no conocen los procesos mentales que soportan el comportamiento leal del cliente, por lo tanto, estas empresas no podrán influir en el comportamiento al no tener el conocimiento real de la causa de lealtad (Delgado, 2004; Odin, Odin \& Valette-Florence, 2001).

El segundo enfoque, lealtad como actitud, la define como un sentimiento de unión o afecto hacia los empleados de una compañía, productos o servicios (Jones \& Sasser, 1995). Se considera la fidelidad como una actitud que supone que diferentes sensaciones del cliente crean un apego individual al servicio (Setó, 2003). Esta visión centra toda la atención en la comprensión de las estructuras mentales, emocionales y de conocimiento como antecedentes del comportamiento (Delgado, 2004), ya que la actitud tiene un carácter predictor y precursor de la conducta (Colmenares $\&$ Saavedra, 2007). Se fundamenta en las teorías del comportamiento, que exponen la lealtad como una actitud que debe expresar todos los elementos que la integran: cognitivos, afectivos y conativos
(Dick \& Basu, 1994; Oliver, 1999). La debilidad de esta perspectiva aparece al pretender caracterizar la lealtad, ya que la existencia de actitudes favorables y compromisos manifiestos por parte del consumidor no garantizan la elección y adquisición del producto o servicio (Colmenares \& Saavedra, 2007).

El tercer enfoque surge como una dimensión integradora o actitudinal-comportamental. Las aportaciones de Day (1969) supusieron un punto de inflexión en la conceptualización del término, al poner de manifiesto la necesidad de incluir un componente actitudinal al comportamental, asumiendo que fidelidad es un constructo multidimensional. Este enfoque entiende la lealtad como la relación entre la actitud relativa hacia una entidad y el comportamiento repetido de compra, como expone Setó (2003). En consecuencia, y tal y como afirma Llorens (2011), Dick y Basu (1994) proponen un modelo que supone el más importante antecedente del modelo de Oliver (1999), el cual ha sido el más reconocido y el que más análisis posteriores ha inspirado. Estos autores realizan una combinación del concepto de actitud con el de repetición de compra, estableciendo en cada uno de estos, dos niveles (alto y bajo). Esta combinación tiene como resultado cuatro situaciones: 1. lealtad (fidelidad verdadera); 2. lealtad latente (clientes potenciales a ser fieles); 3. lealtad espuria (falsa fidelidad) y 4 . sin lealtad (clientes desertores).

De igual forma, Dick y Basu (1994) estudian los antecedentes de la actitud basados en los modelos tradicionales, dividiendo los antecedentes en tres grupos: los cognitivos, los afectivos y los conativos. Por estos motivos, Oliver (1999) ratificado por autores como Yuksel, Yuksel y Bilim (2010), afirma que los clientes se vuelven fieles primero en un sentido cognitivo, seguido de un sentido afectivo, posteriormente conativo y finalmente en forma de comportamiento, es decir, el proceso conlleva cuatro fases: 1 . fase de lealtad cognitiva; 2 . fase de lealtad afectiva; 3 . fase de lealtad conativa; 4. fase de lealtad como acción.

Setó (2003) recoge un enfoque más: la lealtad cognitiva. Esta perspectiva define a los clientes fieles como aquellos que recompraron una marca, considerando solamente esa marca, y no buscaron 
información relacionada a la misma. Este autor plantea que hay investigadores que entienden la lealtad bien como "la primera elección" entre alternativas o bien cuando determinada marca o servicio se convierte en la primera en la mente del consumidor cuando necesita tomar una decisión, como qué comprar o dónde ir.

$\mathrm{Al}$ igual que la conceptualización, las herramientas de medición de la lealtad también han sido tema de debate. En este sentido, basándonos en los enfoques estudiados anteriormente, surgen un número importante de medidas, las cuales pueden dividirse en actitudinales y actitudinales-comportamentales (Delgado, 2004) o en actitudinal o afectiva y conductual (Gil, Berengue, González-Gallarza \& Fuentes, 2007).

Las medidas realizadas dentro del enfoque comportamental son medidas posteriores a la acción, es decir, a la compra del servicio (Colmenares \& Saavedra, 2007). Algunas de las medidas más utilizadas son: tasas de recompra, frecuencia de compras, intensidad de compra... Como crítica a este tipo de medidas se encuentra que desde esta perspectiva no se explota el significado psicológico de la fidelidad y no permite distinguir entre la lealtad verdadera y la falsa lealtad (Setó, 2003).

Las medidas realizadas dentro del enfoque actitudinal miden el grado de compromiso que el cliente adquiere con la empresa. Son consideradas como mediciones anteriores a la acción, es decir, son indicadores de causa (Colmenares \& Saavedra, 2007). En esta aproximación, la medida más aceptada por la literatura es la propuesta por Zeithaml, Berry y Parasuraman (1996). Como críticas se encuentran que los instrumentos utilizados son muy parecidos a los empleados en la evaluación de la satisfacción y con escaso espacio de tiempo entre uno y otro, provocando una sobrevaloración (Mazursky \& Geva, 1989), y que estas medidas asumen una correspondencia positiva entre intenciones y comportamientos, no siendo siempre soportada en estudios empíricos.

Tal y como recogen diferentes autores (Chaudhuri \& Holbrook, 2001; Delgado, 2004) estas medidas son combinaciones de mediciones actitudinales con comportamentales para complementar los análisis, por lo que parecen ser las más apropiadas para valorar todos los componentes del constructo fidelidad.

Respecto al método que se debe emplear para la evaluación de la fidelidad en clientes Ball, Simoes y Machas (2004) proponen que la lealtad entendida desde una perspectiva comportamental puede ser medida simplemente con técnicas de observación, mientras que desde el enfoque actitudinal puede medirse a través de cuestionarios.

Finalmente, como se ha podido comprobar en diferentes sectores, las medidas para evaluar la fidelidad del cliente se pueden clasificar en tres tipos. Sin embargo, aunque se ha evaluado la fidelidad en el sector deportivo, mediante instrumentos que recogen el enfoque comportamental (Pinillos, 2004; Triadó \& Aparicio, 2004), y el enfoque actitudinal (Bodet, 2012; Wei, Hung, Yang \& Jui, 2010), en ningún caso se ha procedido a examinar y validar una escala específica en español en dicho sector. Por esta razón, el objetivo de este trabajo fue adaptar y validar la escala de intenciones futuras de comportamiento en usuarios de servicios deportivos de Zeithaml et al. (1996).

\section{Método}

\section{Participantes}

La muestra se compuso de 2181 usuarios de servicios deportivos, de las cuales el $64.1 \%$ eran varones y el $35.9 \%$ eran mujeres, con una edad media 27.77 \pm 14.95 . El 21.2\% tenía estudios universitarios, el $6.8 \%$ manifestó no poseer estudios, un $56.5 \%$ había cursado primaria y/o secundaria y el 15.5\% poseía el título de bachiller. La frecuencia semanal de práctica era para el $58.6 \%$ de ellos de entre dos y tres veces por semana y un $31.2 \%$ afirmó realizar práctica deportiva 4 o más veces por semana. Finalmente, el tiempo de práctica medio fue de 94.93 \pm 45.64 minutos.

\section{Instrumento}

El instrumento utilizado fue la Escala de Intenciones de Comportamiento de Zeithaml et al. (1996), 
traducida y adaptada a servicios turísticos por Setó (2003). Se trata de una batería de 13 ítems utilizada para poder medir un amplio rango de intenciones de comportamiento. Los autores, tras un análisis factorial exploratorio obtuvieron como resultado la existencia de cinco dimensiones a partir de los trece ítems: Fidelidad, Disposición al Cambio, Sensibilidad al Precio y Comportamiento de Queja en su vertiente Interna y Externa. La escala oscilaba entre 1 (nada probable) y 7 (totalmente probable). En el cuestionario adaptado por Setó (2003), se procedió a sustituir las expresiones agencias y servicios turísticos por organizaciones deportivas y/o servicios deportivos, obteniéndose de esta forma la escala resultante.

\section{Procedimiento}

El trabajo de campo se realizó mediante un cuestionario autoadministrado con presencia del encuestador. Antes de proceder a la recogida de datos, se pidió permiso a los responsables de las diferentes organizaciones que participaron en el estudio. Posteriormente, se solicitó a los participantes que lo cumplimentaran de forma voluntaria y que consultaran cualquier duda que tuvieran con los ítems. Todos los participantes fueron informados de la confidencialidad de los datos y aceptaron contestar al cuestionario. La administración del cuestionario se desarrolló en todos los casos sin incidencias destacables, siendo el tiempo invertido en la realización del mismo de unos 15 minutos

Análisis de datos

Se llevó a cabo el análisis de las propiedades psicométricas de la escala con el paquete informático SPSS.20. En primer lugar se realizó un análisis de los descriptivos (media, desviación estándar, asimetría, curtosis y coeficiente de correlación corregido ítem-total). A continuación se procedió a realizar los análisis factoriales tanto el exploratorio (AFE) como el confirmatorio (AFC).

Previamente al análisis de estimación de los modelos, se calculó el coeficiente de Mardia, para asegurar la presencia de normalidad multivariada en los datos obtenidos. A continuación se efectuó un AFC con el programa AMOS 20.0. Se utilizó el procedimiento de ajuste del modelo de máxima verosimilitud. Para evaluar la bondad del ajuste se revisaron diez indicadores: estadístico ji cuadrado $\left(\chi^{2}\right)$; la razón entre $\chi^{2}$ y el número de grados de libertad $\left(\chi^{2} / g l\right)$; los índices de ajuste parciales de carácter absoluto: GFI y AGFI. Así como los ín-

TABLA 1

Media (M), desviación estándar (DE), asimetría, curtosis, correlación ítem-total (R IT-c) y alfa si algún ítem es eliminado (a sin item)

\begin{tabular}{lcccccc}
\hline & $\mathrm{M}$ & $\mathrm{DE}$ & Asimetría & Curtosis & $\mathrm{R}$ IT-c & $\boldsymbol{a}$ sin ítem \\
\hline Contaré & 5.37 & 1.22 & -0.574 & 0.084 & 0.496 & 0.687 \\
Recomendaré & 5.44 & 1.21 & -0.566 & -0.161 & 0.51 & 0.685 \\
Animaré & 5.4 & 1.26 & -0.628 & 0.042 & 0.469 & 0.689 \\
Consideraré & 5.17 & 1.26 & -0.503 & -0.075 & 0.422 & 0.694 \\
Más & 5.19 & 1.43 & -0.6 & -0.054 & 0.359 & 0.7 \\
Menos & 2.83 & 1.58 & 0.829 & -0.047 & -0.028 & 0.748 \\
Otro & 3.24 & 1.69 & 0.421 & -0.689 & 0.117 & 0.733 \\
Precios & 4.61 & 1.54 & -0.191 & -0.515 & 0.321 & 0.705 \\
Dispuesto & 3.92 & 1.53 & -0.07 & -0.459 & 0.375 & 0.698 \\
Cambiaré & 4.37 & 1.66 & -0.287 & -0.615 & 0.327 & 0.704 \\
Experiencia & 4.65 & 1.6 & -0.337 & -0.641 & 0.447 & 0.688 \\
Problema & 3.66 & 1.82 & 0.23 & -0.977 & 0.384 & 0.697 \\
Reclamaré & 4.95 & 1.61 & -0.383 & -0.737 & 0.416 & 0.692 \\
\hline
\end{tabular}

Fuente: elaboración propia 
dices de ajuste incremental: NFI, TLI, CFI e IFI. Finalmente, los índices de ajuste de carácter parsimonioso: RMR y RMSEA. Además con el objeto de seguir las indicaciones de Byrne (2001) se adjuntó el criterio de información de Aiken (AIC) y el índice de validación cruzada esperada (ECVI).

Finalmente, se calculó la fiabilidad del instrumento resultante mediante el coeficiente alfa de Cronbach, así como la validez discriminante. Para esto, se han aplicado tres procedimientos diferentes: cálculo de correlaciones entre factores, estimación de modelos alternativos y construcción de los intervalos de confianza de la correlación de factores al 95\% de confianza.

\section{Resultados}

En la Tabla 1 se muestran los estadísticos descriptivos de los ítems. Los índices de asimetría y curtosis son próximos al valor cero y por debajo del valor 1.96. Dos ítems (Menos y Otro) no cumplen el criterio de correlación corregido ítem-total, su eliminación supone un incremento en el valor de alfa de Cronbach $(\alpha)$. Estos resultados permiten la utilización de técnicas factoriales. La fiabilidad de la escala $\alpha=0.777$.
Para conocer la estructura factorial de la escala se realizó un análisis factorial exploratorio (AFE) sobre los 11 ítems resultantes, por el método de extracción de componentes principales y posterior rotación VARIMAX. Previo al análisis, se calculó la medida de adecuación muestral de Kaiser-MeyerOlkin (KMO) y el test de esfericidad de Bartlett. El índice $\mathrm{KMO}$ mostró un valor de 0.82 y el test de Bartlett resultó estadísticamente significativo $\left(\chi_{55}^{2}\right.$ $=7275.632 ; p<0.001)$, lo que llevó a concluir que la aplicación del análisis factorial resultaba pertinente. La estructura dimensional resultante está conformada por tres factores (lealtad, sensibilidad al precio y respuesta) que conjuntamente explican un $65.89 \%$ de la varianza (Tabla 2).

Para comprobar que la escala sigue la estructura factorial esperada, se llevó a cabo un AFC. En primer lugar, se constató la existencia de normalidad multivariada en los datos obtenidos, mediante el coeficiente de Mardia que fue de 16.304 que, de acuerdo con Bollen (1989), es inferior a $p(p+2)$, siendo p el número de variables observadas.

El modelo teórico hipotetizado resultante del AFE, compuesto por 3 factores y 11 ítems, obtuvo resultados dispares. Los índices de ajuste GFI $=$ 0.9; $\mathrm{CFI}=0.916$ e IFI $=0.918$ ofrecieron valores

TABLA 2

Estructura factorial rotada, comunalidades, autovalores, alfa de Cronbach y porcentaje de varianza explicada por cada factor

\begin{tabular}{lcccc}
\hline & 1 & 2 & 3 & Extracción \\
\hline Contaré & 0.869 & & & 0.773 \\
Recomendaré & 0.895 & & & 0.819 \\
Animaré & 0.846 & & & 0.73 \\
Consideraré & 0.784 & & & 0.685 \\
Más & 0.635 & & & 0.558 \\
Cambiaré & & 0.762 & & 0.627 \\
Experiencia & & 0.731 & & 0.597 \\
Problema & & 0.811 & & 0.66 \\
Reclamaré & & 0.556 & & 0.449 \\
Precios & & & 0.762 & 0.733 \\
Dispuesto & & 0.758 & 0.617 \\
\% Varianza explicada & & 10.008 & 65.892 \\
Autovalor & 36.945 & 18.939 & 1.101 & \\
alfa de Cronbanch & 4.064 & 2.083 & 0.607 & 0.777 \\
\hline
\end{tabular}

Fuente: elaboración propia 
excelentes. Por el contrario, el resto de los índices presentaron valores inadecuados $(\mathrm{AGFI}=0.839$; $\mathrm{NFI}=0.883 ; \mathrm{TLI}=0.888 ; \mathrm{RMR}=0.231 ; \mathrm{RM}-$ $\mathrm{SEA}=0.102 ; \mathrm{AIC}=176.993 ; \mathrm{ECVI}=0.885 ; \chi^{2}=$ 126.993). El cociente $\chi^{2} / g$ l presentó un valor discreto $\left(\chi^{2} / g l=3.097\right)$. Por tanto, fue necesario modificar el modelo anterior. La reespecificación se efectuó con base en los siguientes criterios: (1) la significatividad de las cargas factoriales, (2) la información proporcionada por la matriz residual y (3) los índices de modificación ofrecidos por el programa. El resultado fue la eliminación del ítem Experiencia.

Se llegó a un modelo reespecificado compuesto por 10 ítems agrupados en 3 factores (Figura 2). La mayor parte de los índices de ajuste propuestos para este nuevo modelo son correctos $(\mathrm{GFI}=0.939$; $\mathrm{AGFI}=0.894 ; \mathrm{NFI}=0.933$; TLI $=0.948$; CFI $=0.963 ;$ IFI $=0.964 ;$ RMR $=0.119 ;$ RMSEA $=$ $0.074 ; \mathrm{AIC}=113.362 ; \mathrm{ECVI}=0.567 ; \chi^{2}=67.362 ;$ $\left.\chi^{2} / g l=2.105\right)$.

La fiabilidad del instrumento final se midió con el coeficiente alfa de Cronbach dando un valor de 0.761 .

La validez discriminante de la escala se ha contrastando por tres vías diferentes. La primera es calcular la matriz de correlaciones entre los ítems de la escala, existiendo correlación significativa, positiva y moderada entre todos los factores que

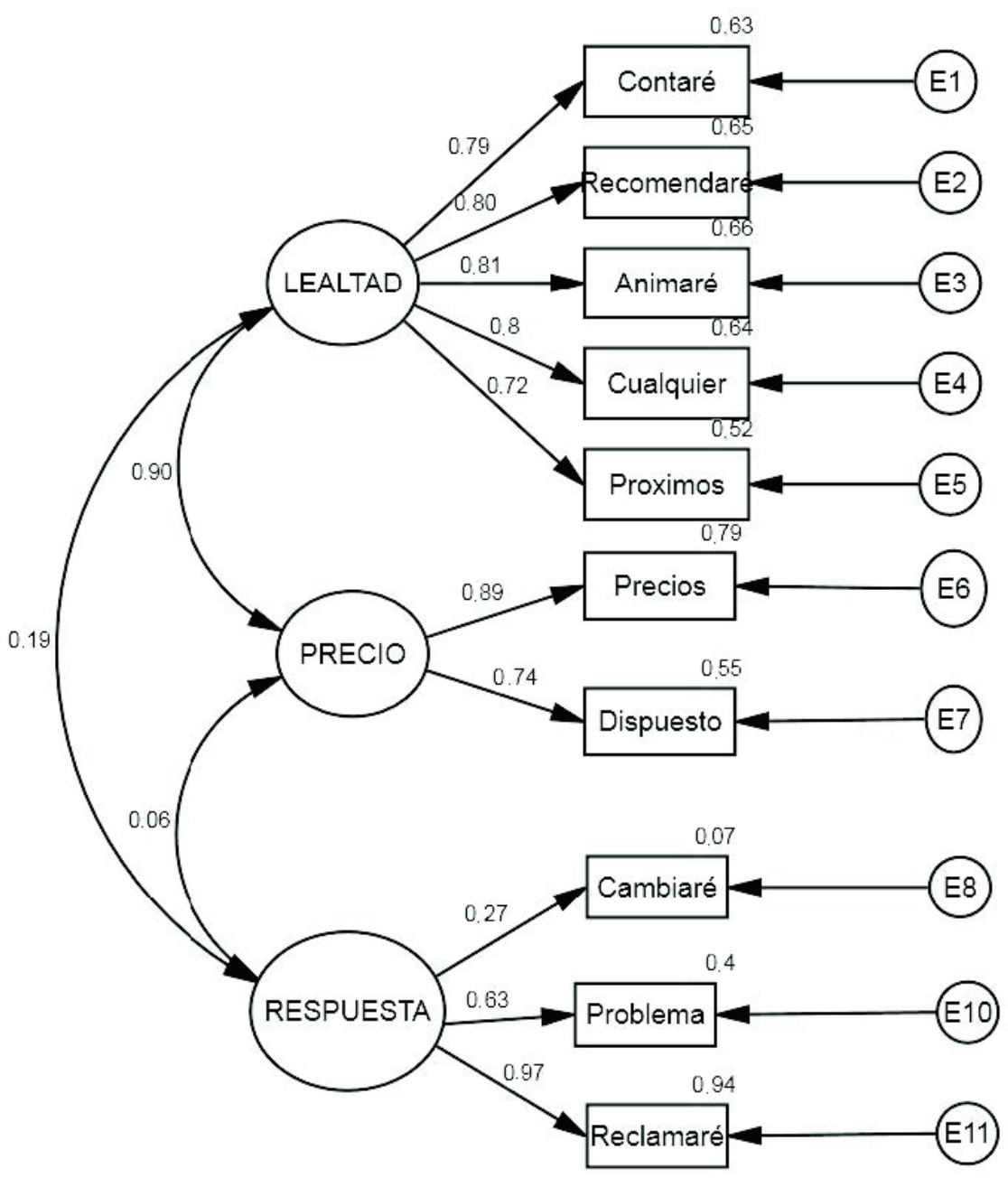

Figura 2. Modelo modificado

Fuente: elaboración propia 
TABLA 3

Correlación entre los factores resultantes, fiabilidad en la diagonal

\begin{tabular}{lccc}
\hline & Lealtad & Precio & Respuesta \\
\hline Lealtad & $(883)$ & $0.456^{* *}$ & $0.119^{* *}$ \\
Precio & & $(607)$ & $0.102^{* *}$ \\
Respuesta & & & $(607)$ \\
\hline
\end{tabular}

** La correlación es significativa al nivel 0.01 (bilateral).

Fuente: elaboración propia

componen la escala, lo que demostró este tipo de validez (Tabla 3).

Actualmente, se utiliza una versión modificada del procedimiento anterior. Se trata de estimar modelos alternativos y realizar con cada uno un test de diferencias de ji cuadrado. En la Tabla 4 se comprueba como la diferencia entre los ji cuadrado fue siempre significativa, por lo que las dimensiones de la escala de intenciones futuras de comportamiento de usuarios de servicios deportivos fueron significativamente diferentes entre sí, corroborando la validez discriminante.

La tercera y última de las vías consiste en calcular las correlaciones posibles entre los factores y construir los intervalos de confianza de las correlaciones entre todas las dimensiones. Los resultados, también han mostrado este tipo de validez, ya que ninguno de los intervalos de confianza de esas correlaciones contiene el valor 1 al 95\% de confianza.

\section{Discusión}

El objetivo de este artículo fue el de adaptar y validar la escala de Zeithaml et al. (1996) de Intenciones Futuras de Comportamiento en usuarios de servicios deportivos, traducida al español por Setó (2003). En primer lugar, se procedió al aná- lisis cuantitativo de los ítems. Este tratamiento tiene como fin garantizar que tengan la capacidad de poner de manifiesto las diferencias existentes entre los individuos. Por ello, el objetivo es conseguir un grupo de ítems que maximice la varianza del test, seleccionando aquellos con un elevado poder de discriminación, alta desviación típica, y con puntuaciones medias de respuesta situadas en torno al punto medio de la escala (Bollen \& Long, 1993; Carretero-Dios \& Pérez, 2005; Nunnally \& Bernstein, 1995).

La puntuación media de los ítems estaba en torno al punto medio de la escala, exceptuando dos ítems (Menos y Otro), y la desviación típica fue superior a 1 , lo que demuestra la normalidad de los resultados según Carretero-Dios y Pérez (2005). Los valores de asimetría y curtosis fueron inferiores a 2 lo que indicaba una distribución normal univariada de los datos (Bollen \& Long, 1993). Para calcular la discriminación de los ítems se recurrió al coeficiente de correlación corregido entre la puntuación en el ítem y la total. Se consideraron adecuados valores mayores o iguales a 0.25-0.35 (Nunnally \& Bernstein, 1995). Finalmente, se tomó la decisión de eliminar los dos ítems que se han mencionado anteriormente y que no cumplían con los dos requisitos.

\section{TABLA 4}

Validez discriminante. Test de diferencias de $\chi^{2}$. Intervalos de confianza de las correlaciones entre las dimensiones

\begin{tabular}{lccc}
\hline & Diferencial de $\chi^{2}(\mathrm{gl})$ & $p$ & Intervalo de confianza \\
\hline Lealtad/Precio & $74.716(33)-67.362(32)=7.354(1)$ & 0 & $(0.413-0.499)$ \\
Lealtad/Respuesta & $136.572(33)-67.362(32)=69.21(1)$ & 0 & $(0.065-0.173)$ \\
Precio/Respuesta & $120.931(33)-67.362(32)=53.569(1)$ & 0 & $(0.045-0.157)$ \\
\hline
\end{tabular}

Fuente: elaboración propia 
Alberto Nuviala Nuviala, Alberto Grao-Cruces, María Rosario Teva-Villén, Raquel Pérez-Ordás, Jerónimo García-Fernández, Ainara Bernal-García

Una vez que los ítems seleccionados pasaron los filtros iniciales, el objetivo siguiente era comprobar si estos empíricamente se agrupaban. En este momento, la meta es explorar la estructura interna de la escala, su dimensionalidad (Elosua, 2003). La escala se construyó alrededor de cinco factores: fidelidad, disposición al cambio, sensibilidad al precio y comportamiento de queja en su vertiente interna e externa. Para comprobar la agrupación se utilizó el AFC. El procedimiento de rotación utilizado fue VARIMAX. Se optó por este, debido al interés teórico de separar, en la medida de lo posible, los factores resultantes (Carretero-Dios \& Pérez, 2007).

Previo a una correcta aplicación del AFE, es necesario contrastar que los ítems deben encontrarse relacionados entre sí. Por ello, es necesario realizar antes de la aplicación del análisis el cálculo de unos estimadores que aseguren que la matriz de correlaciones es la apropiada (Cortina, 1993), siendo las pruebas de elección la de esfericidad de Bartlett y el índice KMO. El resultado de esta prueba fue positivo.

El resultado del análisis estadístico de los ítems y el AFE fue la existencia de tres factores que explican el $65.89 \%$ de la varianza, alrededor de los cuales se agrupaban los ítems: lealtad, sensibilidad al precio y respuesta. El primer factor reúne los cinco primeros ítems que coinciden exactamente con la dimensión "lealtad" propuesta por Zeithaml et al. (1996) y Setó (2003). En el segundo factor, cargan los dos siguientes ítems que se corresponde con la dimensión que ZeithamI et al (1996) etiquetan como "pagar más" y vuelve a coincidir con los resultados de Setó (2003). Por último, el tercer factor aglutina los cuatro último ítems, igual que Setó (2003), que corresponde a las dimensiones que el instrumento original denomina comportamiento de queja en su vertiente externa e interna.

Para comprobar la estructura factorial de la escala conformada por 5 factores y 22 ítems, se llevó a cabo un AFC. El modelo inicial propuesto está muy próximo a cumplir con los requisitos establecidos por Bollen (1989) sobre el número de ítems por factor. Solamente el segundo factor no cumple este requisito al estar compuesto por dos ítems. De la misma forma, el tamaño de la muestra supera los requisitos establecidos por diversos autores según los cuales el número mínimo estaría en 200 sujetos cuando el número de ítems es de 3 por factor o variable latente (Anderson \& Gerbing, 1984) e incluso superior si el número de ítems decrece por variable latente (Marsh, Balla \& McDonald, 1988).

Los parámetros fueron estimados mediante el método de máxima verosimilitud, al cumplirse los dos supuestos de normalidad. En el caso de la univariada, como se ha visto anteriormente, los índices cumplen con las recomendaciones de Bollen y Long (1993). Según Bollen (1989) existe normalidad multivariada si el coeficiente de Mardia es inferior a $\mathrm{p}(\mathrm{p}+2)$, donde $\mathrm{p}$ es el número de variables. Teniendo en cuenta que en este estudio existían 11 variables y el coeficiente de Mardia provisto por el programa AMOS fue igual a 16.304 , se puede afirmar la existencia de normalidad multivariada.

Para evaluar la adecuación del modelo sometido a prueba se optó por la valoración conjunta de un grupo de índices. Los resultados obtenidos en el modelo inicial no aportan resultados óptimos en algunos de los índices (AGFI, NFI, TLI y RMR), asî como para el cociente $\chi^{2} / g l$. Por su parte el criterio de información de Aiken (AIC) mostró un valor muy alto y el índice de validación cruzada esperada (ECVI) obtuvo un resultado próximo a 1. Fue necesario corregir el modelo hasta obtener resultados aceptables. Dicha reespecificación se efectuó con base en los criterios propuestos por Rial, Varela, Abalo y Lévy (2006). El modelo final estuvo compuesto igualmente por 3 dimensiones y 10 ítems, presentado unos índices de ajuste correctos. Así los índices de bondad de ajuste del modelo GFI, NFI, TLI, CFI e IFI exhibieron valores por encima de 0.9, y RMSEA exhibió un valor por debajo del criterio de 0.08. Solamente los índices AGFI (0.894) y RMR (0.119) presentaron valores no aceptables. A pesar de estos dos resultados, el conjunto de los índices de ajuste del modelo llevaron a la decisión de aceptar el modelo siguiendo los criterios de Arbuckle (2007). De igual manera, los índices AIC y ECVI, así como el cociente $\chi^{2} / g l$, mostraron valores menores, lo que significa un mejor ajuste del modelo (Weston \& Gore 2006). 
La validez discriminante de la escala viene expresada por el contraste entre los diferentes factores que la componen. Si son realmente distintos los conceptos que la conforman y al mismo tiempo están relacionados, es entonces cuando se puede hablar de este tipo de validez (Lehmann, Gupta \& Steckel, 1999). Para garantizarla, se han calculado de diversas formas. La primera y tradicional, consiste en correlacionar los factores que componen la escala y comprobar que su relación sea significativa, positiva y moderada. Los resultados obtenidos dan pie para afirmar este tipo de validez. En la actualidad se comprueba a través de otras dos vías. La primera es la propuesta por Burnkrant y Page (1982), que trata de estimar modelos alternativos de tal forma que en cada uno de ellos se introduce como restricción que la correlación entre cada par de dimensiones es igual a 1, y realizar con cada uno un test de diferencias de ji cuadrado para comparar los modelos con el fin de evaluar si eran significativamente diferentes. Los resultados han probado como la diferencia entre los ji cuadrado fueron siempre significativas, por lo que las dimensiones de la escala de intenciones futuras de comportamiento de usuarios de servicios deportivos fueron diferentes entre sí, corroborando la validez discriminante

La tercera y última de las vías consiste en calcular las correlaciones posibles entre los factores y construir los intervalos de confianza de las correlaciones entre todas las dimensiones. Los resultados, también han mostrado este tipo de validez, ya que ninguno de los intervalos de confianza de esas correlaciones contiene el valor 1 al 95\% de confianza (Anderson \& Gerbing, 1984).

En conclusión, los resultados permiten presentar una herramienta capaz de conocer las intenciones futuras de comportamiento de usuarios de servicios deportivos. Tras el AFC, se ha obtenido un cuestionario reducido compuesto por tres dimensiones y diez ítems, lo que supone una reducción de un 23.08\% de los ítems y una pérdida centesimal en el coeficiente alfa de Cronbach (0.016). Estos resultados llevan a afirmar que se trata de un instrumento fiable y válido, lo que lo acredita como útil para la gestión y la investigación.

\section{Referencias}

Anderson, J. C., \& Gerbing, D. W. (1984). The effect of sampling error on convergence, improper solutions, and goodness-of-fit indices for maximum likelihood confirmatory factor analysis. Psychometrika, 49(2), 155-173.

Arbuckle, J. L. (2007). Amos16.0 User's Guide. Chicago, IL: SPSS Inc.

Ball, D., Simoes, P., \& Machas, A. (2004). The role of communication and trust in explaining customer loyalty: An extension to the ECSI model. European Journal of Marketing, 38(9), 1272-1293.

Bodet, G. (2012). Loyalty in sport participation services: An examination of the mediating role of psychological commitment. Journal of Sport Management, 26(1), 30-42.

Bollen, K. A. (1989). Structural equations with latent variables. New York: John Wiley y Sons.

Bollen, K. A., \& Long, J. S. (1993). Testing structural equation models. Newbury Park, CA: Sage.

Burnkrant, R. E., \& Page, T. J. (1982). An examination of the convergent, discriminant and predictive validity of Fishbein's behavioral intention model. Journal of Marketing Research, 19(4), 526-550.

Buttle, F., \& Burton, J. (2002). Does service failure influence customer loyalty. Journal of Consumer Behavior, 1(3), 217-227.

Byrne, B. M. (2001). Structural equation modeling with AMOS. Basic concepts, applications, and programming. Londres: LEA.

Carretero-Dios, H., \& Pérez, C. (2005). Normas para el desarrollo y revisión de estudios instrumentales. International Journal of Clinical Health Psychology, 5(3), 521-551.

Carretero-Dios, H., \& Pérez, C. (2007). Standards for the development and the review of instrumental studies: Considerations about test selection in psychological research. International Journal of Clinical Health Psychology, 7(3), 863-882.

Colmenares, O., \& Saavedra, J. L. (2007). Aproximación teórica de la lealtad de marca: enfoques y valoraciones. Cuadernos de Gestión, 7(2), 69-81.

Cortina, J. M. (1993). What is coefficient alpha? An examination of theory and applications. Journal of Applied Psychology, 78(1), 98-104. 
Alberto Nuviala Nuviala, Alberto Grao-Cruces, María Rosario Teva-Villén, Raquel Pérez-Ordás, Jerónimo García-Fernández, Ainara Bernal-García

Chaudhuri, A., \& Holbrook, M. B. (2001). The chain of effects from brand trust and brand affect to brand performance: The role of brand loyalty. Journal of Marketing, 65(2), 81-94.

Chen, S. C., \& Quester, P. G. (2006). Modeling store loyalty: perceived value in market orientation practice. Journal of Services Marketing, 20(3), 188-198.

Day, G. (1969). A two-dimensional concept of brand loyalty. Journal of Advertising Research, 9, 29-36.

Delgado, E. (2004). Estado actual de la investigación sobre lealtad de marca: una revisión teórica. Revista de Dirección, Organización y Administración de Empresas, 30, 16-24.

Dick, A. S., \& Basu, K. (1994). Customer loyalty: Toward an integrated conceptual framework. Joumal of Academy of Marketíng Science, 22(2), 99-113.

Elosua, P. (2003). Sobre la validez de los tests. Psicothema, 15(2), 315-321.

Gil, I., Berengue, G., González- Gallarza, M., \& Fuentes, M. (2007). Segmentando clientes a partir del valor del servicio. Una aproximación en el contexto de la relación entre empresas. Cuadernos de Economía y Dirección de Empresas, 31, 31-66.

Jacoby, J., \& Chestnut, R. W. (1978). Brand loyalty measurement and management. New York: John Wiley \& Sons.

Jones, T. O., \& Sasser, W. E. (1995). Why satisfied customers defect? Harvard Business Review, 73(6), 88-99.

Lehmann, D. R., Gupta, S., \& Steckel, J. H. (1999). Marketing research. New York: Addison-Wesley.

Llorens, J. (2011). La lealtad de los aficionados al futbol. Una explicación en base al valor percibido de marca de su equipo y su nivel de implicación. Tesis doctoral, Universitat Jaume, Castellón de la Plana, España.

Marsh, H. W., Balla, J. R., \& McDonald, R. (1988). Goodness-of-fit indexes in confirmatory factor analysis: The effect of sample size. Psychological Bulletin, 103(3), 391-410.

Mazursky, D., \& Geva, A. (1989). Temporal decay satisfaction-purchase intention relationship. Psychology E3 Marketing, 6(3), 211-227.
Nunnally, J. C. \& Bernstein, I. J. (1995). Teoría psicométrica. Madrid: McGraw-Hill.

Odin, Y., Odin, N., \& Valette-Florence, P. (2001). Conceptual and operational aspects of brand loyalty: An empirical investigation. Journal of Business Research, 53(2), 75-84.

Oliver, R. L. (1999). Whence consumer loyalty? Journal of Marketing, 63, 33-44.

Pinillos, J. (2004). Winning the retention battle: librando la batalla por la retención. Investigación y Marketing, 83, 39-42.

Rial, A., Varela, J., Abalo, J., \& Lévy, J. P. (2006). El Análisis Factorial Confirmatorio. En J. P. Lévy \& J. Varela (Coords.), Modelización con estructuras de covarianzas en ciencias sociales. Temas esenciales, avanzados y aportaciones especiales (pp.119-154). A Coruña: Netbiblo.

Setó, D. (2003). La fidelidad del cliente en el ámbito de los servicios: un análisis de la escala intenciones de comportamiento. Investigaciones Europeas de Dirección y Economía de la Empresa, 9(2), 189-204.

Triadó, X., \& Aparicio, P. (2004). El tipo de cuota como variable de fidelización de usuarios en entidades deportivas: un reto actual de consecuencias futuras. Investigación y Marketing, 83, 31-38.

Wei, P., Hung, H., Yang, H., \& Jui, Y. (2010). Examination of the influence of service quality on membership renewal in fitness centers in San Francisco Bay Area. Journal of Service Science, 3(2), 13-24.

Weston, R., \& Gore, P. A. (2006). A brief guide to structural equation modeling. The Counseling Psychologist, 34(5), 719-751.

Yuksel, A., Yuksel, F., \& Bilim, Y. (2010). Destination attachment: Effects on customer satisfaction and cognitive, affective and conative loyalty. Tourism Management, 31(2), 274-284.

Zeithaml, V. A., Berry, L., \& Parasuraman, A. (1996). The behavioral consequences of service quality. Journal of Marketing, 60(2), 31-46. 


\section{ANEXo 1}

Cuestionario definitivo extraído del Análisis Factorial Confirmatorio

\begin{tabular}{cll}
\hline \multirow{2}{*}{ Factor } & \multicolumn{1}{c}{ Ítem abreviado } \\
\hline \multirow{3}{*}{ Lealtad } & Contaré & Contaré aspectos positivos sobre el club deportivo a otras personas \\
\cline { 2 - 3 } & Recomendaré & Recomendaré el centro deportivo a cualquiera que busque mi consejo \\
\cline { 2 - 3 } & Animaré & Animaré a mis amigos y familiares a hacer actividades deportivas en este centro \\
\cline { 2 - 3 } & Consideraré & $\begin{array}{l}\text { Para cualquier servicio deportivo que pudiera necesitar, consideraré este club como } \\
\text { la primera opción }\end{array}$ \\
\cline { 2 - 3 } Mrecio & Más & En los próximos años realizaré más actividades deportivas en este club \\
\cline { 2 - 3 } & Precios & Aunque los precios sean algo más altos permaneceré en este centro deportivo \\
\hline \multirow{3}{*}{ Respuesta } & Dispuesto & $\begin{array}{l}\text { Estoy dispuesto a pagar un precio más alto que los cargados en otros gimnasios por el } \\
\text { servicio que recibo }\end{array}$ \\
\cline { 2 - 3 } & Cambiaré & Cambiaré a centros deportivos, si tengo un problema con el servicio \\
\cline { 2 - 3 } & Problema & $\begin{array}{l}\text { Si tengo un problema con el gimnasio, reclamará a entidades externas como la Aso- } \\
\text { ciación de Consumidores y Usuarios }\end{array}$ \\
\cline { 2 - 3 } & Reclamaré & Si tengo un problema con el servicio, reclamaré al director del centro deportivo \\
\hline
\end{tabular}


